\title{
The general Design of Tester System Based on ARM of Fuse
}

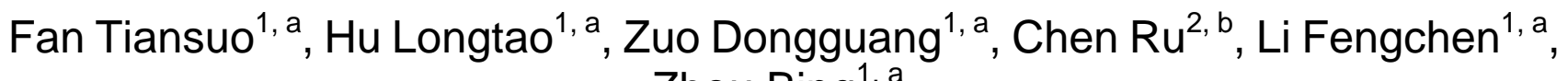 \\ Zhou Bing ${ }^{1, a}$ \\ ${ }^{1}$ The Rocket Force engineering university ${ }^{\text {dep }}$, Xi 'an 710025, China; \\ ${ }^{2}$ The Rocket Force Command college, Wuhan 430012, China. \\ a523098802@qq.com, ${ }^{b} 1475411493 @ q q . c o m$
}

Keywords: fuse safety, fuse tester, The ARM microcontroller, rapid, detection.

\begin{abstract}
Fuse is the key part of detonate control, and it's state determines the weapon whether can accord to the scheduled model of initiation to achieve the objective of the damage or not. To achieve the best effect of damage, the fuse detector are used generally to detect whether the performance of fuse whether is normal or not. Aiming at present fuse system tester's problem of difficult generation, troublesome operation and bad environmental adaptability, a general design method of fuse Tester which is based the ARM is proposed in this paper. Test results show that the method can realize the detection for some types of fuse, and it contains advantages of simply detection, friendly interface, flexible man-machine interaction, small size, carrying easily and simple maintenance, and it meets the demand of operations and training of troops.
\end{abstract}

\section{Introduction}

Fuse plays a very important role in the weapon system as fusing control components, in order to ensure the reliability of Fuse, Fuse test work is very necessary. Modern Fuses contain data signal processing, interference and jamming, target distance measurement, control detonated, targeting a variety of functions target and error information processing, which put forward higher requirements to the fuse system, fuse system should first cover the content of fuse test coverage, meanwhile it must also ensure test accuracy controllable. ${ }^{[1]}$. In order to solve practical problems of a machine testing and other existing multi-fuse tester can't achieve generalized to meet the fuse outfield experiment and troops operational needs, this paper universally designs and researchs the fuse tester system which is based on ARM microcontroller.

This paper puts forward the general design scheme of fuse test instrument based on ARM system through to analyze the empty fuse, trigger fuse and electromechanical fuse common fuse test requirement, and all the parts of the tester for fuse, function realization and so on were introduced in detail analyzed.

\section{2. fuse test content analysis.}

Fuse testing usually include:

2.1 check the zero position of the fuse

By checking Fuse zero position to judge the working status of fuse, the judgment of fuse also is the solution to the state insurance to protect state, if the solution is in the state guarantee, the fuse before the test must be reset, it is the insurance status, to ensure that fuse Test security.

The check of the zero position of the fuse is usually realized by measuring the break through of the path. When the pathway for the connected state (resistance is less than or equal to $10 \mathrm{ohms}$ ), fuse in release state; when the pathway for the disconnected state (resistance value is equal to or more than $20 \mathrm{~m}$ Ohm), fuse in insurance status [2].

2.2 simulation of fuse solution to protect signal 
When the fuse is tested, fuse tester $t$ applied simulation solution of environmental protection directives to the fuse operation timing simulation solutions to protect instruction while measuring levels of safety fuse is normal lift.

When the fuse works, taking into account the safety factor, usually set between the power supply and fuse terminal multi-level insurance, power and pyrotechnics isolated, only when the fuse normal operation, the insurance only chronological progressively lifted, signals are usually security solutions voltage signal or contact signal.

When the solution is applied to the analog command to fuse protection must take security measures to ensure that the warhead in a safe condition. Pyrotechnics can simulate real alternative to the pyrotechnic or take other measures against short-circuit protection pyrotechnics, ensure safety fuses during testing.

\section{3 fuse performance test}

According to the time sequence, the fuse is released gradually, and tests the performance. The performance test of the fuse is usually judged by the output voltage or waveform of the fuse [3].

\section{function of fuse tester.}

According to the fuse test content analysis, fuse tester includes tester should have a self-test and verification capabilities, determine the types of fuzes and fuze zero state detection, fuze and fuze function simulation solutions ensure performance testing and other functions:

Fuse tester should carry out self-test and verification before tested to ensure that the fuse tester is in proper working condition to diagnose and to ensure safe and fault of tester, in order to ensure the accuracy and validity of test results. When no escorts letter, the tester is in self-test, all functions and hardware connection status can be checked by using a cable connection. Using standard signal generator signal to fuse tester to complete measurement of the tester voltage and time accuracy of the test, the tester should have automatically determine and display the self-test and calibration results.

To judge the type and position of the fuse. Ueing Wheatstone bridge to measure resistance value which is between the fuze and tester connection the cable core wire by measuring the resistance value judgment during the on-off, in order to determine the type of fuze and the corresponding zero state. Zero state also known for Fuze initial state of fuze initial state is arming) state monitoring and control to determine fuze is arming, and tracking. Fuse tester can through the cable core wire, circuit breaker guide display on the initial state of a signal lights by indicating lights out to judge the insurance status of fuze. Resistance value should be less than $10 \mathrm{ohm}$ for access and lighting. Zero state representation; resistance values greater than $10 \mathrm{~m}$ ohms circuit and off, which represents non null state. In this way, the design can avoid the false identification of the simple on-off identification, and ensure the reliable identification of the fuze type.

Simulation of the function of the fuze in the solution protection. Through the software of ARM to achieve control, make it simulate and issue a release signal and follow by issue of detonating signal and a reset signal operation instructions, the tester can transmit analog transmit signal (power supply) and analog generator ready, simulated far solution on the fuze for integrated logic control electricity to electricity, and other functions.

The tester can achieve real-time testing. Through switching action at all levels before and after the change of the contact state real-time monitoring. to realize the insurance status monitoring records, tracking and control, real-time record, in turn, to fuse solution to protect state at all levels. The of tester followe removed insurance signals before and after the execution of value change information accurately record key contact point voltage of fuse. Also it can realize the safe storage, to ensure that the information loss after power off will not occur. Its function diagram is showd in figure1. 


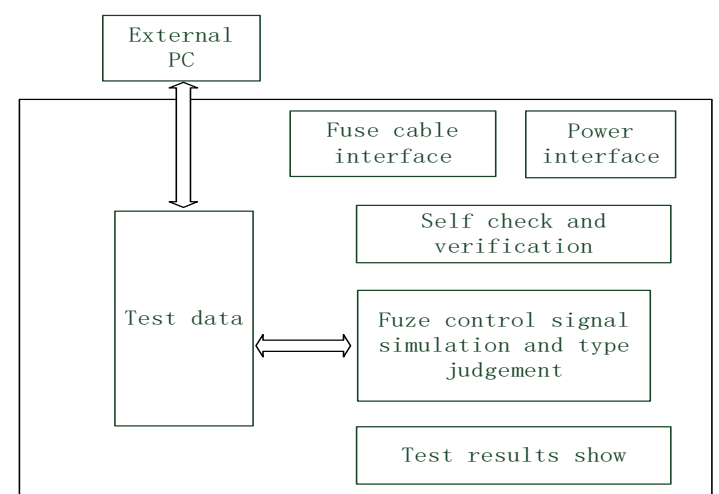

Fig. 1 Schematic diagram of fuse testing instrument function

\section{The design of fuse tester.}

\subsection{Control scheme selection}

Measurement and control system has many kinds of architecture, modern control system basically is mixed analog to digital intelligent measurement and control system, according to the different types of processors are based on IPC computer measurement and control system based on embedded processor (MCU, DSP, FPGA and arm type) of embedded control system. According to the requirements of the fuse tester under high and low temperature, hot and humid, portability and reliability. Based on IPC computer measurement and control system is difficult to meet the above requirements, so the scheme of measurement and control the embedded control system framework [4].

Embedded processor has a lot of kinds, like phyletic, MCU, DSP, FPGA (SOPC), ARM processor type, etc. SCM is limited by its processing speed and peripherals, does not apply to the design requirements; FPGA (SOPC) need to build on its own processor core and peripherals, high workload, reliability cannot be verified in a short period of time, also not suitable for this design applications; DSP and ARM processor strengths and the hardware design on the DSP chip by adder, more suitable for signal processing, and ARM processor used to compare the rich peripherals, more suitable for signal processing and control integration products, and architecture (M4 series of ARM processor has built-in floating-point hardware by unit, or even better than a lot of DSP in the signal processing ability. So, choose the architecture (M4 series of ARM processor as the control unit of the fuze tester controller [5].

3.2 Tester composition

According to testing requirements, will be the fuze based on ARM's fuse tester could be divided into three part :control unit, display unit and power supply unit.

Control unit mainly contains ARM processor, signal conditioning circuit, driver circuit, communication interface and etc, as showed in figure 2, it completes the power-up and de-fuse protection, signal conditioning. voltage resistance testing and communications functions [6].

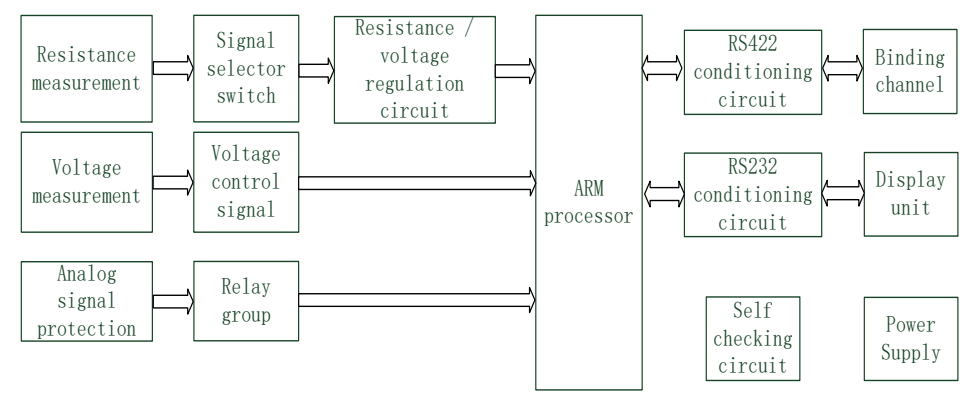

Fig. 2 Principle design block diagram of measurement and control unit 
Fuse tester makes control unit as the core, after completing the self-test, turns on the fuse zero level check and sends fuze simulation solution to protect instruction, fuze performance test, the real-time display of test results and the test results are stored [7].

Self-test circuit provides standard voltage to meet the requirements of the self-test, resistance signals to meet the needs of the self-test unit; multiple resistance measurement signal through the signal selection switch, the final choice to give way to resistance or voltage signal conditioning circuits, the output voltage signal by AlD channel from the ARM acquisition and processing, the other a few road voltage measurement signals are attenuated by the voltage conditioning circuit into the same number of channels by the ARM acquisition and processing.

ARM control via relay output control corresponding voltage by GPIO. Control unit sets test parameters of fuze by RS422, exchanges the information with display unit through the RS232.

The display unit completes test results in real time fuze display, prints and stores in the pilot testing program. Composition is showed in figure 3.

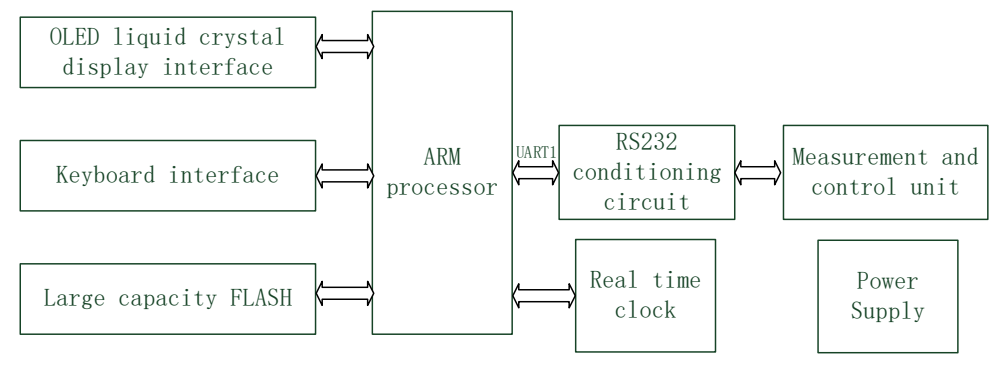

Fig. 3 Principle block diagram of display unit

According to the requirement of size, weight, reliability and data storage needs, The display unit using the rchitecture way of ARM + OLED LCD + keyboard + peripheral driver to meet the fast start, miniaturization and high reliability requirements. Built-in real time clock, backup battery can be replaced, and there can set the time according to demand. Taking into account the requirements of reliable operation of the device in a variety of environments, so a mechanical keyboard interacts with the user, the corresponding measurement data through OLED LCD module to graphically display the interface, measurement data automatically determine the voltage waveform has qualified to judge, for artificial Analyzing reference. FLASH large-capacity built-in file system, the measurement data stored in chronological order in the file system, and the interface can be exported via USB.

Power module mainly realizes AC/DC converter to provide the required DC power supply for the tester and the tested fuse.

\section{Key Technology Solutions}

\section{1 security design}

Security design is an important key technology of test instrument, its safety device for fuze safety and the safety tester have the vital significance. Therefore the design of fuze tester in the course of the development of security is based on the functional design focuses on performance. Intends to adopt the following measures to ensure the safety of fuze tester, tester level of safety monitoring design, testers, confirmed the safety of the design, the safety current design sensitivity.

The fuze for perfect fuze under test and test process for personnel to site operation, the security of the testing process is very important. General tester test safety design is the key. Safety testing process mainly includes the following aspects:

(1) fuze safety test status. Corresponding conductor cable connection relations decided to fuze working mode and test mode for normal work mode or safety. Tester to test of the fuze types must be in safety test mode, avoid into the working mode of the work was delayed blasting fuse initiating explosive device, the fuse to be measured and safety accidents caused by the tester. A few type of fuze setting safety test status to ensure accurate; 
(2) fuze is the key solution to protect signal is carried out. Fuze signal fuze is executed far solutions cover instruction, therefore, must be confirmed before the signal far solution the fuse the normal state, such as, the fuse is in a state of safety testing, fuze of initiating explosive device access plug has been pulling, fuze types and recognition to the fuze type, etc. If appeared above abnormal signal far solution may result in unsafe state;

(3) fuze power solutions for power supply. Solution of fuze signal for high-power electric signal, supply initiating explosive device, high power solution to protect the power supply in fuze abnormal working state will result in initiating explosive device, dangerous for fuze and testers to be tested.

In view of the above safety analysis, general test instrument designs the following measures to ensure test security:

(1) special test cable, safety test socket and plug the wrong design. Fuze used special test cable, cable plug cable connects to the fuse, the other end of the test cable tester for access to the test socket. Multiple test socket tester, corresponding to different fuze, the test socket for the cable core definition based on increased root core, three test socket and the corresponding digital cable conductor completely consistent, the definition of digital definition respectively after different fuze, different core (head) splice different hole (core). Beside each socket on the tester body engraved with the name of the corresponding fuse, in case the operator to insert the plug will be measured when the fuze types make confirmation with the corresponding socket type, as a safety test to confirm.

(2) the corresponding design of the first stage power supply and the type of fuze. Fuze level powers supply from the tester for different control respectively. Fuze level 1 powers supply and fuse type correspondence, tester output fuze level power supply outputs and tester is to identify corresponding to the fuze.

When the tester is recognition to the access of the fuze target fuze, is confirmed with a debugger via a serial port for telemetry simulation solution module corresponding instructions, telemetry and protect module only through simulation solution to the target of the fuze power relay, level of power supply to the appropriate output fuze test socket conductor, the target of fuze power supply channels, there is no other power supply, above can't give target fuze power supply, power supply channel is as long as the access of the fuze and recognition to the fuze types inconsistent on the fuze can't electricity. Once again achieved a safety test measures;

(3) initiating explosive device connected to the plug. Fuze has important safety joint should be pulled up processing, to ensure that the fuze of driving energy current is not connected to the initiating explosive device circuits of initiating explosive device. Of initiating explosive device access plug parts pictures, when tester self-inspection and fuze types identification confirm the identity of the fuze corresponding status and pictures, if not consistent, shall be promptly removed the initiating explosive device access plug, may continue to test;

(4) the safety current sensitivity design. Despite the above two levels of security design, but still need to avoid in tester testing and personnel safety confirmation link failure and ensure the safety of the tested fuze failure. Tested fuze major hazards is the solution of initiating explosive device, in order to ensure that the test will not cause in the process of gunpowder pull pin system, will give power supply ability of the fuze test power electric control in the primary solution to protect signal required by the current range, below pin pull signal and power, as one of tester security safeguards.

In addition, in the solution of dentsu bao road, all designed to recover current limiting the insurance. Ensure that no large current through the solution to protect channel, avoid explosion point of initiating explosive device.

In summary, the test detected by the security test status, the cable tester one correspondence error protection design, supply and fuse tester corresponds to the type of tester Pyrotechnics access unplug confirmation and security current, etc. multiple links together to ensure the safety of the testing process. Even if the test detected the wrong type fuse tester detect security test status error, there fuzefuze and fuze power signal corresponding to the type of measures tester cables connected correctly. Its safety failure rate is lower than millionth. 


\subsection{Compatibility design of test types of fuze}

In order to meet the test requirements of a variety of fuze, the isolation of the test method of fuze key contacts and signal measurement, to avoid the design to the internal circuit of the fuze don't understand the problem, under the guidance of the software, according to the fuze types into different test procedures, but also to meet the requirements of a variety of fuze test.

In addition, the cable test different fuze test plug only corresponding to the test instrument of fuze in different test socket, ensure the fuze safety.

There are three test socket tester, respectively corresponding multimode / nondelay fuse series, trigger fuze and fuze. Internal power supply in addition to the first level of power supply is shared by the power supply channel. The communication interface, the telemetry voltage and the type of the fuze and the zero position identification are all used in the same channel. Through the above design and Realization of the four types of fuze universal compatibility test, to ensure the safety of the test process.

4.3 anti-interference design

In order to improve the testing instrument anti-interference ability, circuit adopts the following measures: (1) circuit design in power input interface to add EMC filter, digital simulation and separate layout, to ground; (2) the signal line and the power line separation, transmission using shielded cable of weak signal; (3) important and vulnerable to interference signal by photoelectric isolation and relay isolation measures; (4) test instrument power and fuze power isolation design, testing instrument for Fuze will not cause interference to work, the work will not affect the test instrument of power devices in the fuze action; (5) the tester uses the whole shielding technology, prevent the external electromagnetic interference effects on the internal microcontroller; (6) measuring instrument with single chip microcomputer software adopts event driven software architecture design, accident under outside interference is not working properly and also can quickly return to work, not Enter failure mode.

\section{Summary}

The designs of generation of fuse tester system based ARM, meets the fuse tester universal, portable and other design requirements, multi-type target fuse to detect, with good interface, functional, easy to use and functional scalable, easy system maintenance. And provides the field testing and practical application an effective and convenient testing tools, high performance of its own safety, to ensure that the operation of the measurement from the external environment. give the design of test system, development a good reference.

\section{References}

[1] Wang Bing, RUAN Chaoyang. General Technology of System on Chip Based Programmable Fuze ESA Monitor[J]. Journal of Detection \&Control, 2013, 35(3):61-64.

[2] Ma Shaojie, Zhang He. Classification and correction strategy of fixed distance error for air blast Fuze [J]. Journal of Ballistics， 2008， 20(3):75-78.

[3] Wang Shuilian. Development of special purpose fuse testing system [D]. Harbin Institute Of Technology, 2013.

[4] Wang Bing, Mo Jianjun. Knowledge Acquisition System Constructed by C++ Builder[J].JOURNAL OF SYSTEM SIMULATION， 2002, 14(10):1356-1358.

[5] Zhanwen XI, Weirong NIE, Qilei LI. A MEMS Interrupter Mechanism for Fuse Safety \& Arming Device [J].International Conference on Mechanical Engineering and Mechanics, 2009, 3:51-56 
[6] Fang Shaojun, Li Guolin, Shang Yaling. General Design of the Control Circult for a Contact Airburst Fuze[J]. Journal of Detection \& Control, 2006, 28 (1): 21-24.

[7] Zhang Yajun, Zhang Jianjun, Qi Xihong, Cheng Linlin. Development of automatic detecting instrument for many kinds of fuse circuit board [J]. Mine Warfare \& Ship Self-defence, 2014, 22 (2): 30-33. 